\title{
The importance of wavelength for tight temperature control during $\mu$-laser assisted machining
}

\author{
Ashley Dennis ${ }^{a}$, Saurav Goel ${ }^{a, b^{*}}$, Rajab Al-Sayegh ${ }^{c}$ and William O'Neill ${ }^{d}$
}

aSchool of Aerospace, Transport and Manufacturing, Cranfield University, Bedfordshire, MK43 0AL, UK

bSchool of Engineering, London South Bank University, 103 Borough Road, London SE1 OAA, UK

${ }^{c}$ College of Engineering, Northern Border University, Arar, 91431, Saudi Arabia

IInstitute for Manufacturing, University of Cambridge, 17 Charles Babbage Road, Cambridge CB 30FS, UK

*Corresponding Author: GOELS@LSBU.AC.UK

\section{Abstract}

The area of single point diamond turning of brittle materials like semiconductors and ceramics is significantly benefitted by incorporation of laser assistance. In a new developmental technology that is now recognised as micro-Laser Assisted Machining ( $\mu$-LAM), a laser is shone through a diamond tool to soften the high pressure phase transformed ductile machining phases that in turn allows thermal softening and thereby enables a higher material removal rate during ductile-mode machining. One of the lasers currently used in $\mu$-LAM is the Nd:YAG laser operating at $100 \mathrm{~W}$ (continuous wave) at the wavelength of $1064 \mathrm{~nm}$. Although, this configuration has worked to the benefit of the technology, here we report futuristic developments that will significantly enhance temperature control by selecting a laser wavelength according to the material being machined, allowing tuneable machining properties. The concept is illustrated with sample calculations for $\mu$ LAM of silicon, and it appears to offer better target temperatures, thus enhancing the performance of the $\mu$-LAM process. 


\subsection{Introduction}

In pursuit of overcoming the challenges of machining difficult-tomachine materials, laser assistance during mechanical micromachining has been adopted worldwide. To date, seven major patents (in year 1982 [1], year 2006 [2], year 2007 [3], year 2011 [4], 2014 [5, 6], year 2017 [7] and references therein) have been granted in the US concerning the use of laser assistance during mechanical micromachining. While these patents fall in the category of thermally assisted machining (TAM), of particular interest is the patent on micro-Laser assisted machining ( $\mu$-LAM) [3]. The concept of $\mu$-LAM is quite unique in the sense that the laser beam is passed through the diamond tool itself (Figure 1).

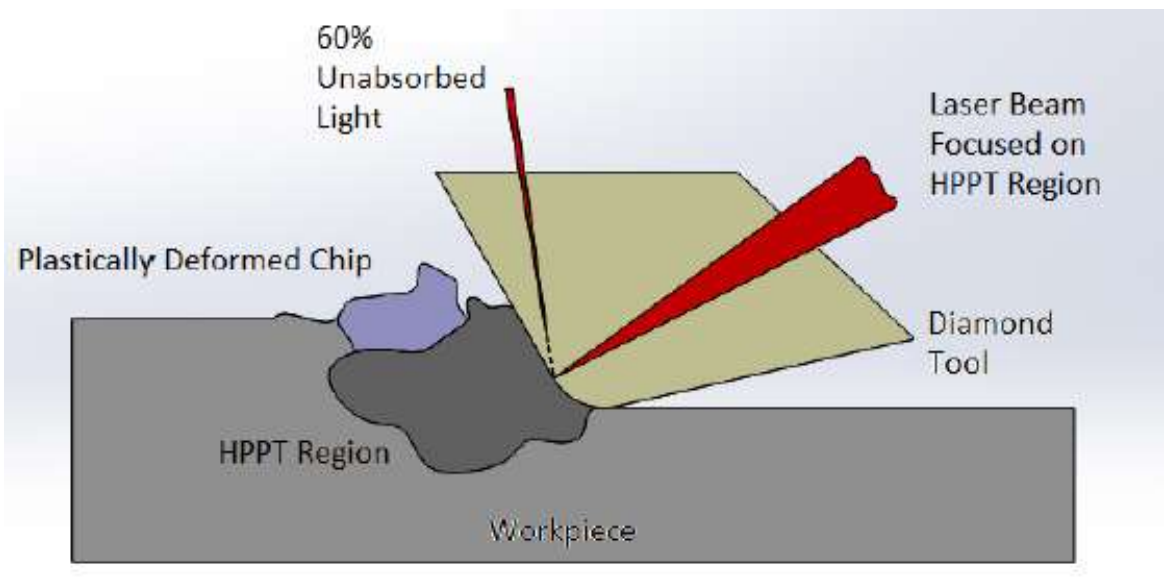

Figure 1 - $\mu L A M$ (Adapted [12])

This approach exploits the fact that the diamond tool is transparent in the near infra-red (IR) region of the spectrum, as are the atmospheric phases of the brittle materials (workpiece of silicon, tungsten carbide etc). However, it is established from the previously published literature on the topic that the amorphous phases generated in the cutting zone are not transparent to the laser. This allows precise and selective heating of the plastically deformed cutting zone where ductile amorphous phases are generated by the virtue of high pressure phase transformation [8]. When compared to other thermally assisted machining processes, the $\mu$-LAM approach avoids problems such as oxidation of the material, thermal damage, as well as other sorts of energy losses due to convection and radiation. Consequently, the absorption of the IR by the highpressure phases (exhibiting a metallic conductive nature) helps to 
achieve higher stock removal rates by improving the achievable brittle-ductile transition cut chip thickness. Reported values of the preferred parameters for laser power are in the range of $8 \mathrm{~mW}$ to $100 \mathrm{~W}$ (of which $40 \%$ is transmitted into the workpiece) and the preferred tip or edge radius of the tool is about $20 \mathrm{~nm}$ to $12 \mu \mathrm{m}$. Other advantages of $\mu$-LAM are reduced surface roughness, which Mohammadi et al (2015) found when turning silicon. More recently, the technique is being proposed to be capable of machining stainless steel almost at the traditional speeds of SPDT thereby being more productive than techniques like ultrasonic elliptical vibration assisted machining (UEVAM) where the speed of cutting needs to be lowered significantly [9].

$\mathrm{Nd}$ :YAG lasers (1064 $\mathrm{nm}$ ) are often used in $\mu$-LAM because of ease of installation as the laser can be taken to the source via a fibre optic cable. The selection of laser wavelength is critical because it determines the amount of heating that is caused in a given material, some materials absorbing more heat at a given wavelength, and some less. It is also noteworthy that the absorptivity of the material changes as a function of material temperature, so the rate of heating changes continuously with time as the material being cut heats up primarily due to laser heating but also due to the cutting heat flux generated in the cutting zone due to plastic deformation of the material. Due to this, most materials display exponential heating so that heating is effectively limited within a given range to avoid extreme heating which would result in melting of the material or combustion. This aspect however, does not seems to be paid attention to in the experimental implementation of micro-LAM and in this article, we share our views on how this additional knowledge of laser-matter interaction may benefit this technology further.

It is to be noted that silicon like most other materials, absorbs more at increased temperatures that can be explained by the relation of its electronic and optical properties. At wavelengths close to 294 $\mathrm{nm}$, however, the absorptivity actually decreases as the temperature increases thus limiting any attainable benefit by microLAM in this regime [10]. We postulate that there exists a specific threshold temperature beyond which no further heating takes place when exposed to laser. It is suggested that this concept could be used to set temperatures for materials cut by $\mu$-LAM, by selecting 
lasers with most appropriate wavelengths so that the energy expenses incurred during laser heating are optimum.

In this article, this concept is illustrated with reference to silicon, which is a well-studied machinable material in ultra-precision machining. In our calculations, we also show that in addition to the heating time that the substrate should take, the depth at which the heating is taking place confirms that the heating penetrates deep enough into the substrate to have a significant contribution.

\subsection{Analysis}

The relevant formulae for the calculations for heating of silicon are shown in this section. A material's surface temperature increase can be calculated according to the formula [11]:

$$
T=T_{0}+\frac{\alpha q t\left(1-R_{t}\right) e^{-\alpha t}}{\rho C_{P}}
$$

where $T$ is the temperature $\left({ }^{\circ} \mathrm{C}\right)$ of a material to be calculated at an initial $T_{0}$ initial temperature $\left({ }^{\circ} \mathrm{C}\right), \alpha$ is the absorptivity $(/ \mathrm{m}), q$ is the power density $\left(\mathrm{W} / \mathrm{m}^{2}\right), t$ is laser dwell time (s), $R_{t}$ is the reflectance $\rho$ is density $\left(\mathrm{kg} / \mathrm{m}^{3}\right)$, and $C p$ is the specific heat capacity $(\mathrm{J} / \mathrm{kg} \mathrm{K})$.

Absorptivity in turn is given by the formula:

$$
\alpha=\frac{4 \pi n(T) k(T)}{\lambda}
$$

where $\alpha$ is the absorptivity $(/ \mathrm{m}), n$ is material's refractive index, $k$ is the extinction coefficient, and $\lambda$ is wavelength of the light (m).

The refractive indices $n$ and $k$ are dependent on the temperature expressed by the formulae [10] as below:

$$
\begin{aligned}
& n\left(T_{0}+\Delta T\right)=n\left(T_{0}\right)\left(1+c_{n} \Delta T\right) \\
& k\left(T_{0}+\Delta T\right)=k\left(T_{0}\right)\left(1+c_{k} \Delta T\right)
\end{aligned}
$$


where $T_{0}$ is base temperature $(300 \mathrm{~K}), \Delta T$ is change in temperature, $\mathrm{cn}$ and $\mathrm{ck}$ represent the change in refractive index with temperature.

Green et al. [10] provided values of refactive index and extinction coefficient for silicon at various wavelengths, and the rate of change for the values (see Appendix 1). The refractive index and the value of extinction coefficient of silicon at a fixed temperature of $300 \mathrm{~K}$ (figure $2 \mathrm{a}$ and figure $3 \mathrm{a}$ ) and the coefficients for temperature dependence (figure $2 \mathrm{~b}$ and figure $3 \mathrm{~b}$ ) are presented. A significant difference in the value of refractive index and extinction coefficient, affecting the absorptivity, can be predicted at elevated temperature. Taking this data, figure 4 was obtained, that shows that the absorptivity of silicon is indeed strongly dependent on temperature. A change of sign in absorbtivity of silicon was noted close to 0.29 to $0.31 \mu \mathrm{m}$ for the calculations performed by assuming the silicon to be heated by $1110 \mathrm{~K}$ (which compares well to a melting temperature of $1140 \mathrm{~K}$ of silicon). 


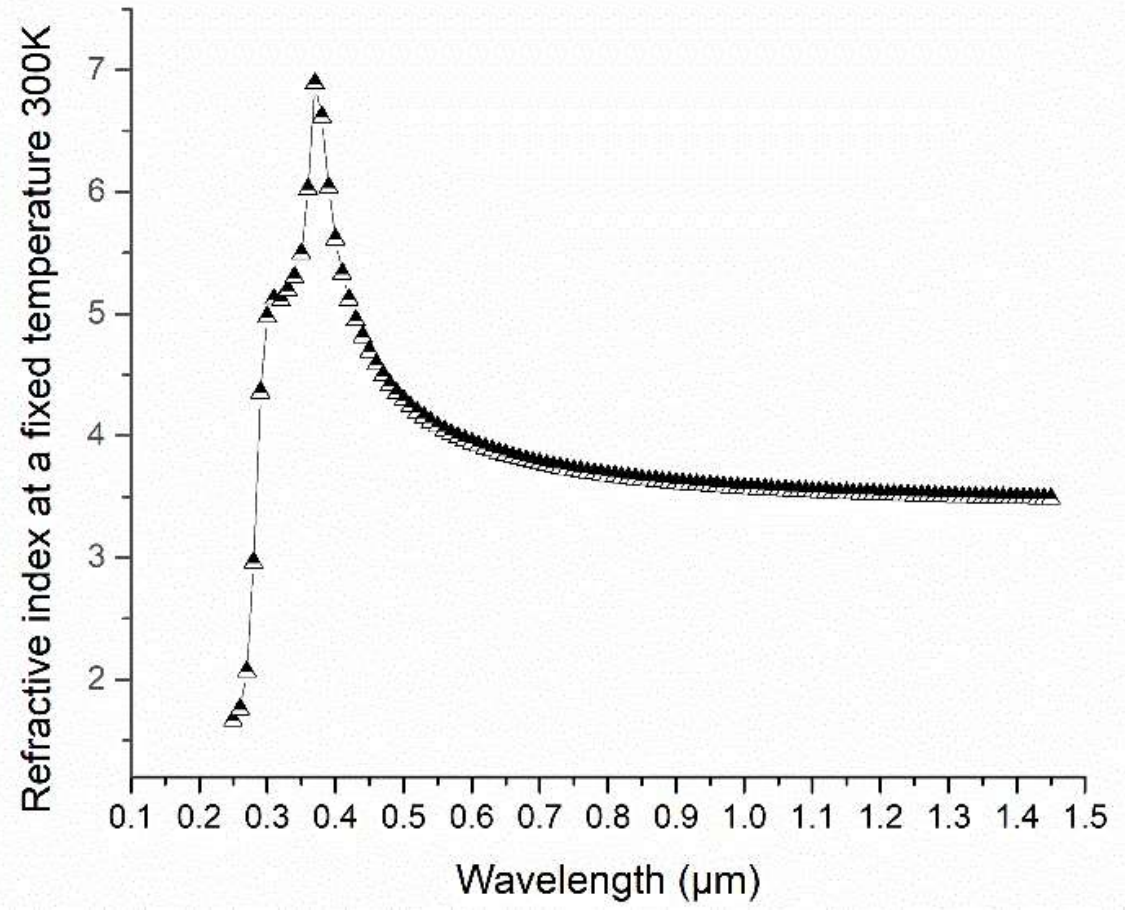

(a)

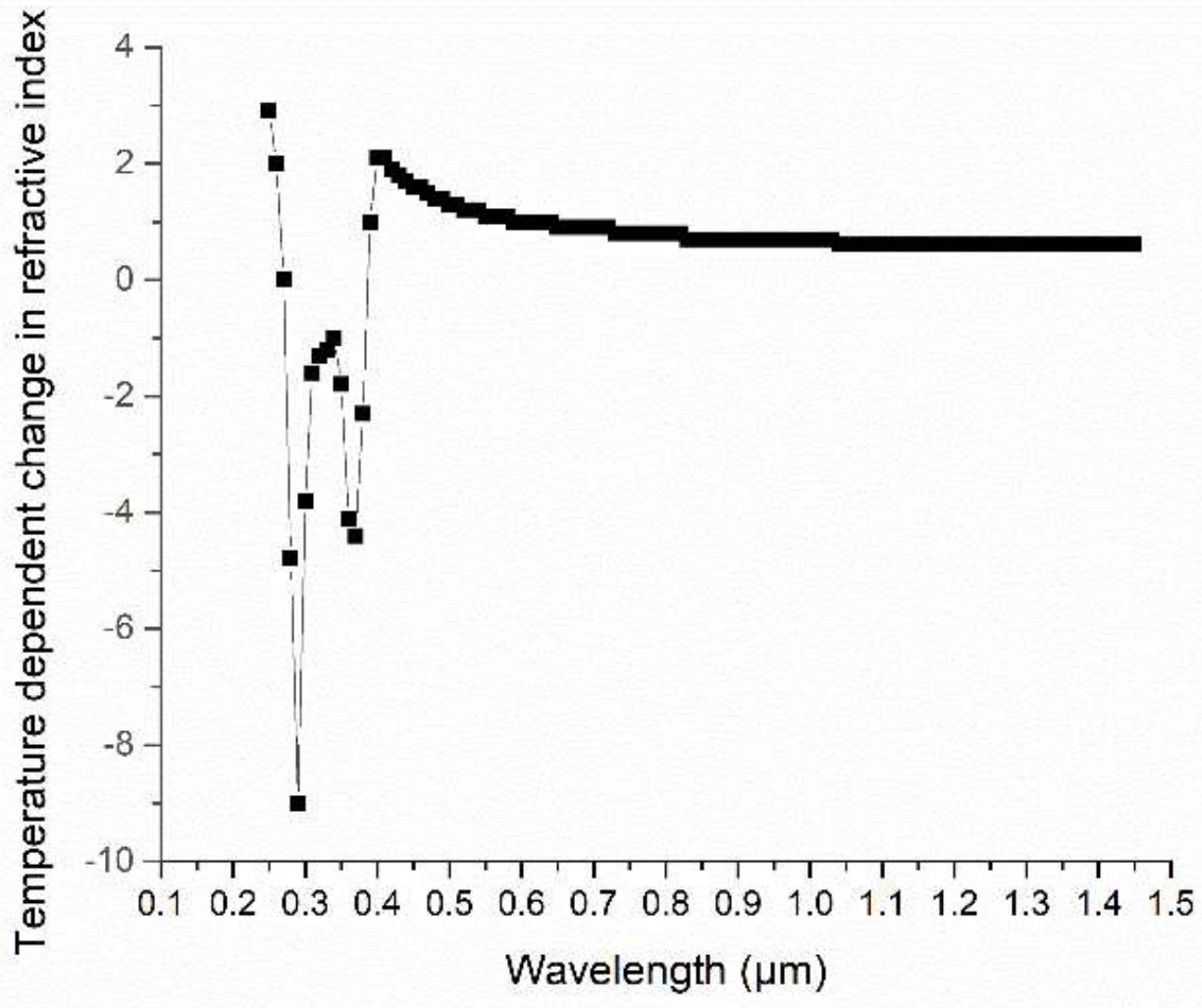

(b)

Figure 2 - Refractive Index (N) values for silicon (Adapted [10]) at (a) a fixed temperature and (b) at a varying temperature 


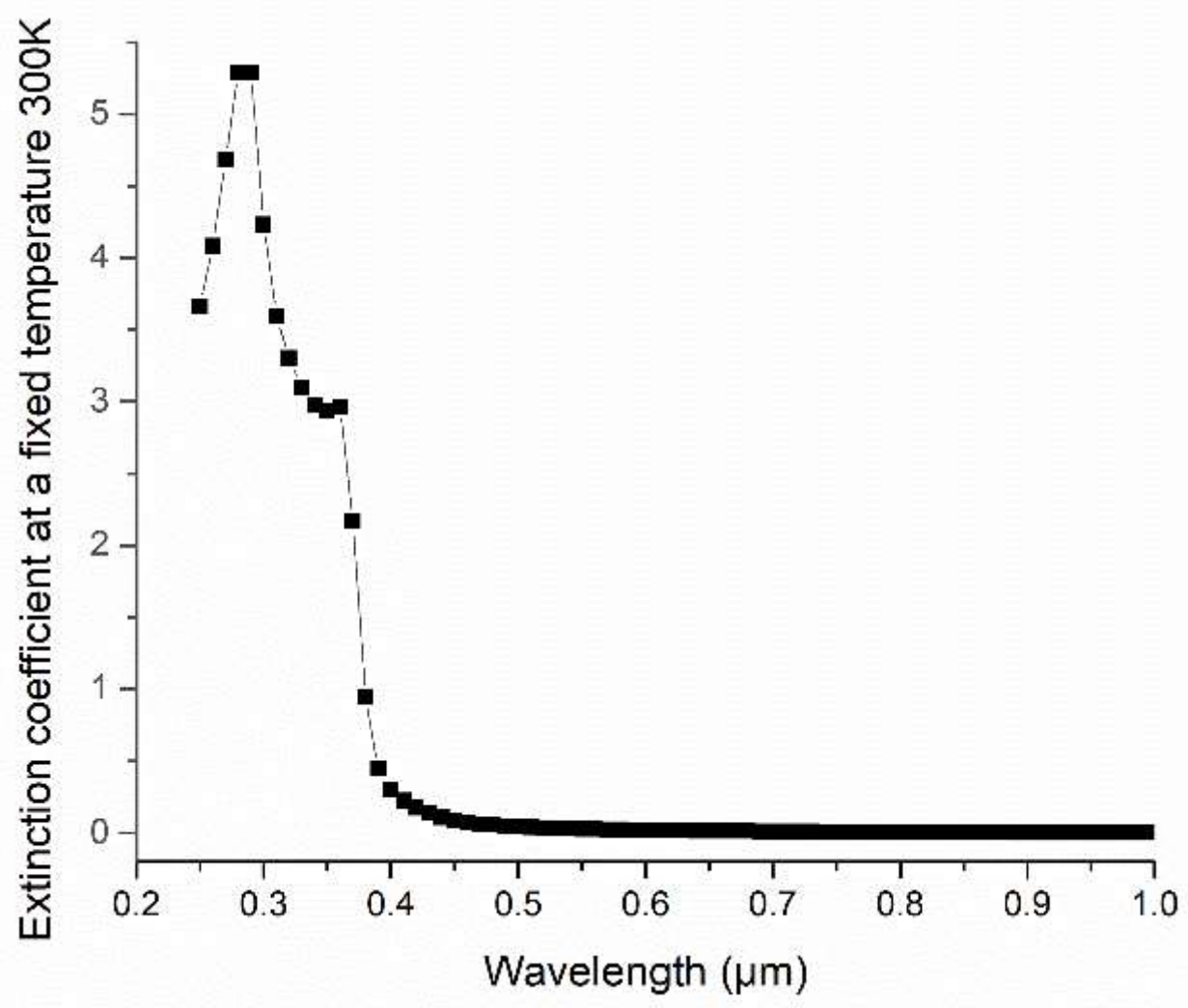

(a)

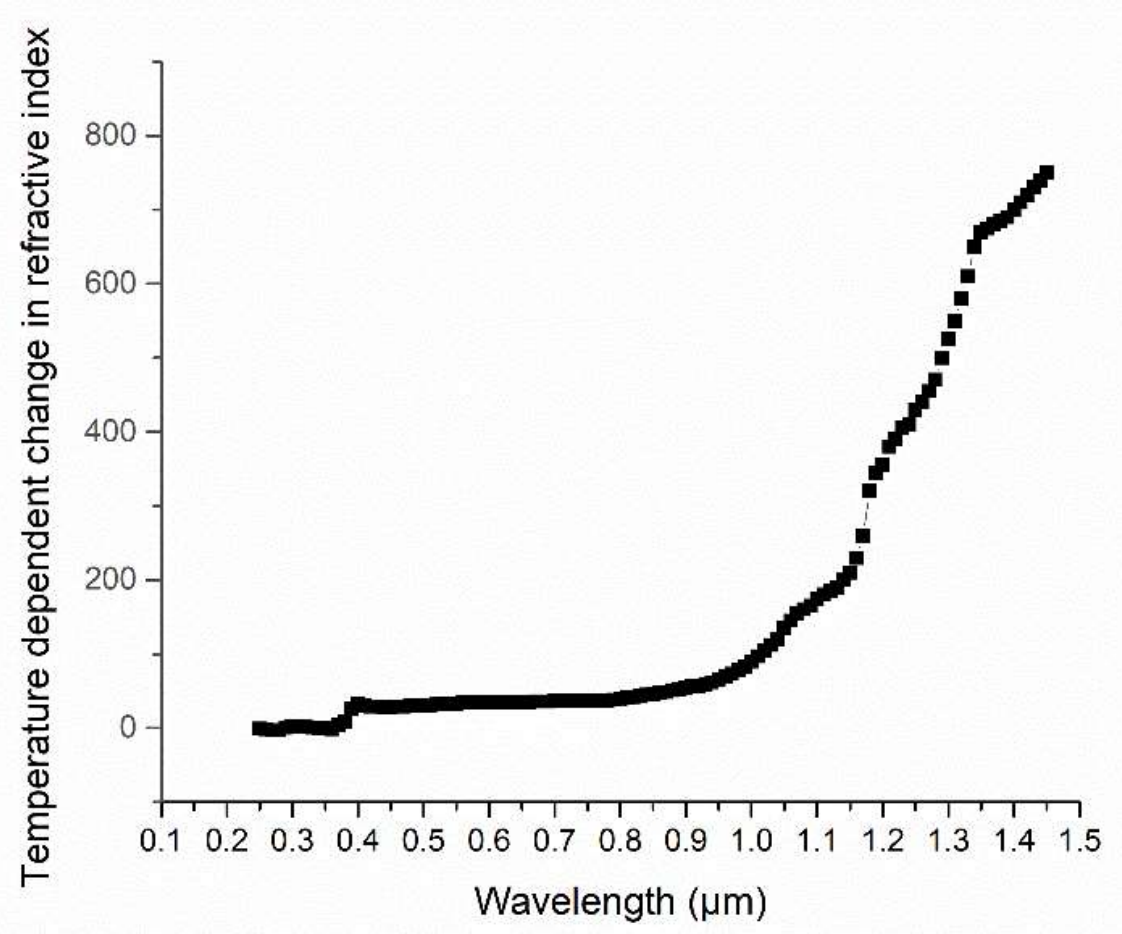

(b)

Figure 3 - Extinction Coefficient (k) values for silicon (Adapted [10]) at (a) a fixed temperature and (b) at a varying temperature 


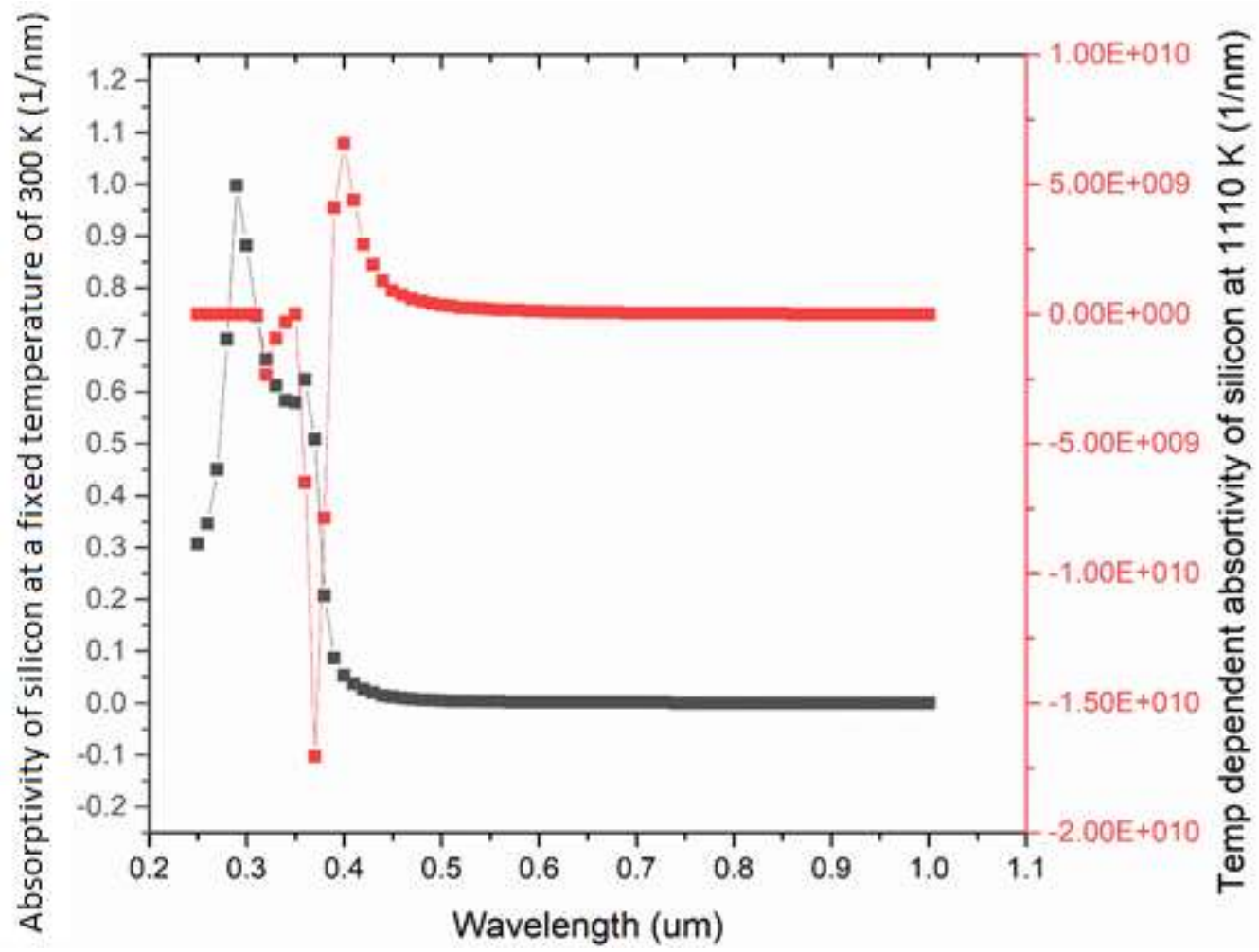

Figure 4 - Comparison of absorptivity of silicon at $300 \mathrm{~K}$ and $1110 \mathrm{~K}$

Formula 1 was then used to calculate the incremental temperature absorbed by silicon (see figure 5) assuming that the heating was done by a $1 \mathrm{~W}$ laser. It is evident that the heating to the target temperature of $1375^{\circ} \mathrm{C}$ occurs within a timespan of about 14 nanoseconds and no further heating occurs thereafter as the absorptivity becomes negligible. 


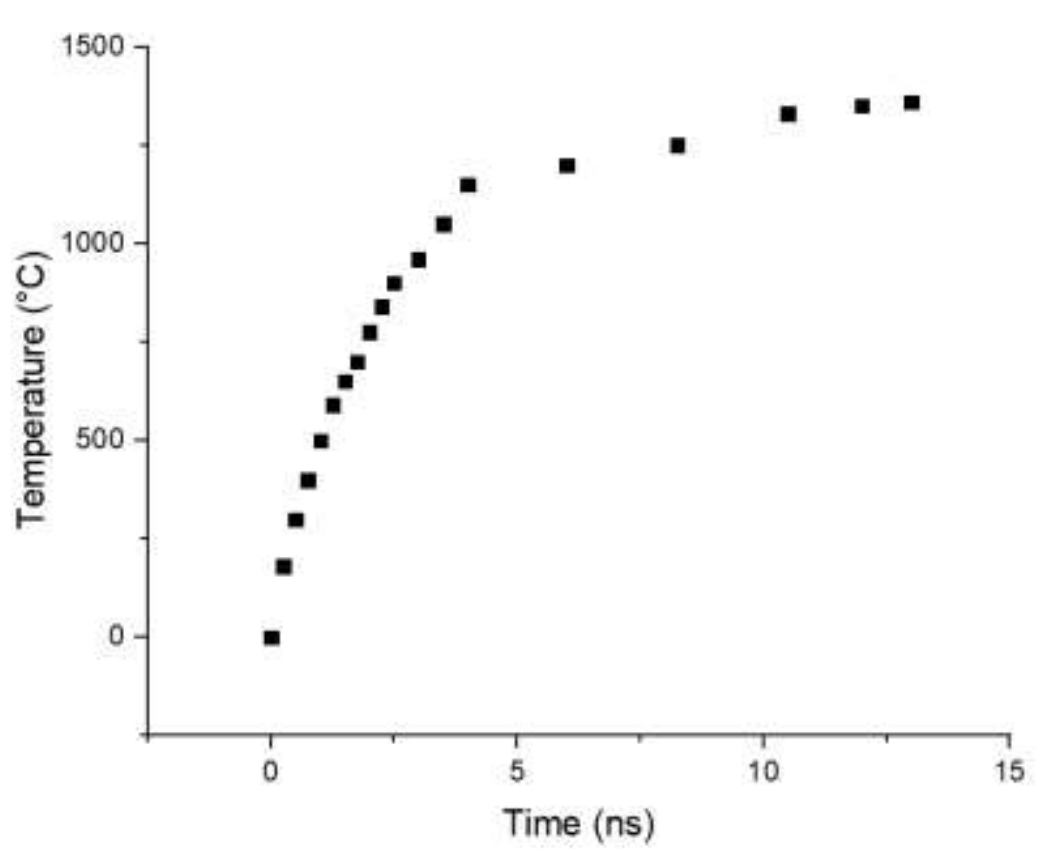

Figure 5 - Numerical Simulation of Heating of Silicon with $1 \mathrm{~W}$, $294 \mathrm{~nm}$ laser

We next focus our attention on the depth of heating which is influential in deciding the depth of material being removed. The depth of absorption is the inverse of absorptivity and is given by the formula:

$l=\frac{\lambda}{4 \pi n k}$

where $\lambda$ is the wavelength $(m), n$ is the refractive index at that wavelength, $\mathrm{k}$ is the extinction coefficient at that wavelength.

The equation reveals that for silicon, the depth of heating at 1375 ${ }^{\circ} \mathrm{C}$ is only about $200 \mathrm{~nm}$. As the depth of heating is small, the conductive heat transfer formulae below were used:

$Q=\frac{k A\left(T_{H O T}-T_{C O L D}\right) t}{d}$

$Q=m \times C_{P} \times T$

where $Q$ is heat transfer, $k$ is thermal conductivity, $C_{P}$ is specific heat capacity, $T_{\text {HOT }}$ is the higher temperature, $T_{\text {COLD }}$ is the lower temperature, $t$ is time and $d$ is distance between the two points, $T$ is temperature 
The parameters used to verify the heating of silicon are shown in table 1:

Table 1 : Heating parameters of silicon $[12,13]$

\begin{tabular}{|l|l|}
\hline Parameter & Value \\
\hline $\mathrm{K}$ & $120 \mathrm{~W} / \mathrm{m} \cdot \mathrm{k}$ \\
\hline $\mathrm{C}_{\mathrm{p}}$ & $750 \mathrm{~J} / \mathrm{Kg} \cdot \mathrm{K}$ \\
\hline $\mathrm{A}$ & $2 \times 10^{-12} \mathrm{~m}^{2}$ \\
\hline $\mathrm{T}_{\text {HOT }}$ & $1410^{\circ} \mathrm{C}$ \\
\hline $\mathrm{T}_{\text {COLD }}$ & $20^{\circ} \mathrm{C}$ \\
\hline $\mathrm{D}$ & $100 \mathrm{~nm}$ \\
\hline $\mathrm{T}$ & $0.56 \mu \mathrm{s}$ \\
\hline $\mathrm{T}$ & $1400^{\circ} \mathrm{C}$ \\
\hline
\end{tabular}

Using the equations and table 1 parameters, it can be shown that within 560 ns (which compares well with typical speed at which the laser moves during micro-LAM, considering the focal spot of the laser and its cutting speed) it is possible to heat a cube of $7 \mu \mathrm{m}$ sides, to the temperature of $1400^{\circ} \mathrm{C}$, indicating that the shallow depth of laser absorption may not be prohibitive.

\section{Conclusions}

This article sheds light on a possible improvement in the area of micro-Laser Assisted Machining ( $\mu$-LAM) which is an advancement to the Single Point Diamond Turning (SPDT) process. As the analysis presented demonstrates, there seems to be potential for controlling temperature in $\mu$-LAM by selection of right laser wavelength according to material's absorptivity. It seems that the temperature in the actual experimentation must be significantly greater than those reported in prior experimental trials $\left(1038{ }^{\circ} \mathrm{C}\right.$, [14]). It appears to be a way forward to achieve a consistent temperature in the cutting zone, by more predictively using the laser parameters. It is difficult to predict the increased advantages that 
the higher temperature would provide, but Ravindra [14] identified from his studies that the optimal temperature is that closest (just below) the melting point achievable, so this technique may further facilitate that approach. It would be desirable to carry out experimental trials in which the effectiveness of the technique could be properly evaluated, although unfortunately there is not yet sufficient experimental information available, using even robust techniques like IR imaging or thermocouples to get the exact assessment of temperature in the cutting zone in $\mu$-LAM.

\section{Acknowledgments:}

The work was carried out in the Centre for Doctoral Training in Ultra-Precision, which is supported by the UKRI via Grants No.: EP/K503241/1 and EP/L016567/1. Part of this work used Isambard Bristol, UK supercomputing service accessed by Resource Allocation Panel (RAP) grant. SG is particularly grateful to the fantastic financial support provided by the UKRI (Grant No. EP/S013652/1, EP/T001100/1 and EP/S036180/1), H2020 (Cost Actions (CA15102, CA18125, CA18224 and CA16235) and EURAMET EMPIR A185 (2018)), Royal Academy of Engineering (Grant No. IAPP18-19l295), and Newton Fellowship award from the Royal Society (NIF\R1\191571).

\section{References:}

[1] R. Komanduri, M. Lee, D.G. Flom, R.A. Thompson, M.G. Jones, R.J. Douglas, Pulse laser pretreated machining, Google Patents, 1982.

[2] J.F. Wu, Y.B. Guu, Laser assisted machining method and device, Google Patents, 2006.

[3] J. Patten, Micro laser assisted machining, Google Patents, 2007.

[4] Y.C. Shin, Laser assisted machining process with distributed lasers, Google Patents, 2011.

[5] Y.C. Shin, Machining apparatus and process, Google Patents, 2014.

[6] N.B. Dahotre, S. Santhanakrishnan, Laser-assisted machining (lam) of hard tissues and bones, Google Patents, 2014.

[7] J.S. Santner, F.M. Sciammarella, S. Kyselica, Laser assisted machining system for ceramics and hard materials, Google Patents, 2017.

[8] H. Mohammadi, D. Ravindra, S.K. Kode, J.A. Patten, Experimental work on micro laser-assisted diamond turning of silicon (111), Journal of Manufacturing Processes 19 (2015) 125-128.

[9] S. Goel, F.D. Martinez, S.Z. Chavoshi, N. Khatri, C. Giusca, Molecular dynamics simulation of the elliptical vibration-assisted machining of pure iron, Journal of Micromanufacturing 1(1) (2018) 6-19. [10] M.A. Green, Self-consistent optical parameters of intrinsic silicon at $300 \mathrm{~K}$ including temperature coefficients, Sol Energ Mat Sol C 92(11) (2008) 1305-1310.

[11] S. Li, L. Li, Radiative transfer modeling for quantifying lunar surface minerals, particle size, and submicroscopic metallic Fe, Journal of Geophysical Research: Planets 116(E9) (2011). 
[12] E. Donovan, F. Spaepen, D. Turnbull, J. Poate, D. Jacobson, Heat of crystallization and melting point of amorphous silicon, Appl Phys Lett 42(8) (1983) 698-700.

[13] G. Benedetto, L. Boarino, R. Spagnolo, Evaluation of thermal conductivity of porous silicon layers by a photoacoustic method, Appl. Phys. A 64(2) (1997) 155-159.

[14] D. Ravindra, Ductile mode material removal of ceramics and semiconductors, Department of Mechanical and Aeronautical Engineering, Western Michigan University, Michigan, 2011, p. 312.

\section{Appendix}

Table 2 - Refractive Indices and Temperature Coefficients for Silicon [11]

\begin{tabular}{|c|c|c|c|c|c|}
\hline$\underset{(\mu \mathrm{m})}{\lambda}$ & $\alpha(/ \mathrm{cm})$ & $n$ & k & $\begin{array}{c}C_{n} \\
(10-4 / \mathrm{K}) \\
(1 / n) \\
(\mathrm{d} n / \mathrm{d} t)\end{array}$ & $\begin{array}{c}C_{\mathrm{k}, \alpha} \\
(10-4 / \mathrm{K}) \\
(1 / k)(\mathrm{d} k / \mathrm{d} t)\end{array}$ \\
\hline 0.25 & 1840000 & 1.665 & 3.665 & 2.9 & -0.9 \\
\hline 0.26 & 1970000 & 1.757 & 4.084 & 2 & -1.5 \\
\hline 0.27 & 2180000 & 2.068 & 4.68 & 0 & 3.1 \\
\hline 0.28 & 2370000 & 2.959 & 5.287 & -4.8 & -3.3 \\
\hline 0.29 & 2290000 & 4.356 & 5.286 & -9 & 0.8 \\
\hline 0.3 & 1770000 & 4.976 & 4.234 & -3.8 & 2.5 \\
\hline 0.31 & 1460000 & 5.121 & 3.598 & -1.6 & 3.2 \\
\hline 0.32 & 1300000 & 5.112 & 3.303 & -1.3 & 1.5 \\
\hline 0.33 & 1180000 & 5.195 & 3.1 & -1.2 & 0.7 \\
\hline 0.34 & 1100000 & 5.301 & 2.977 & -1 & 0.3 \\
\hline 0.35 & 1060000 & 5.494 & 2.938 & -1.8 & 0 \\
\hline 0.36 & 1040000 & 6.026 & 2.966 & -4.1 & -1.4 \\
\hline 0.37 & 737000 & 6.891 & 2.171 & -4.4 & 4.2 \\
\hline 0.38 & 313000 & 6.616 & 0.946 & -2.3 & 9.1 \\
\hline 0.39 & 143000 & 6.039 & 0.445 & 1 & 26 \\
\hline 0.4 & 93000 & 5.613 & 0.296 & 2.1 & 33 \\
\hline 0.41 & 69500 & 5.33 & 0.227 & 2.1 & 31 \\
\hline 0.42 & 52700 & 5.119 & 0.176 & 1.9 & 29 \\
\hline 0.43 & 40200 & 4.949 & 0.138 & 1.8 & 29 \\
\hline 0.44 & 30700 & 4.812 & 0.107 & 1.7 & 28 \\
\hline 0.45 & 24100 & 4.691 & 0.086 & 1.6 & 28 \\
\hline 0.46 & 19500 & 4.587 & 0.071 & 1.6 & 29 \\
\hline 0.47 & 16600 & 4.497 & 0.062 & 1.5 & 29 \\
\hline 0.48 & 14400 & 4.419 & 0.055 & 1.4 & 30 \\
\hline 0.49 & 12600 & 4.35 & 0.049 & 1.4 & 30 \\
\hline 0.5 & 11100 & 4.294 & 0.044 & 1.3 & 31 \\
\hline 0.51 & 9700 & 4.241 & 0.039 & 1.3 & 31 \\
\hline 0.52 & 8800 & 4.193 & 0.036 & 1.2 & 32 \\
\hline 0.53 & 7850 & 4.151 & 0.033 & 1.2 & 33 \\
\hline
\end{tabular}


Draft for J of Micromanufacturing

\begin{tabular}{|r|r|r|r|r|r|}
\hline 0.54 & 7050 & 4.112 & 0.03 & 1.2 & 33 \\
\hline 0.55 & 6390 & 4.077 & 0.028 & 1.1 & 33 \\
\hline 0.56 & 5780 & 4.045 & 0.026 & 1.1 & 34 \\
\hline 0.57 & 5320 & 4.015 & 0.024 & 1.1 & 34 \\
\hline 0.58 & 4880 & 3.988 & 0.023 & 1.1 & 34 \\
\hline 0.59 & 4490 & 3.963 & 0.021 & 1 & 34 \\
\hline 0.6 & 4175 & 3.94 & 0.02 & 1 & 34 \\
\hline 0.61 & 3800 & 3.918 & 0.018 & 1 & 35 \\
\hline 0.62 & 3520 & 3.898 & 0.017 & 1 & 35 \\
\hline 0.63 & 3280 & 3.879 & 0.016 & 1 & 35 \\
\hline 0.64 & 3030 & 3.861 & 0.015 & 1 & 35 \\
\hline 0.65 & 2790 & 3.844 & 0.014 & 0.9 & 35 \\
\hline 0.66 & 2570 & 3.828 & 0.013 & 0.9 & 35 \\
\hline 0.67 & 2390 & 3.813 & 0.013 & 0.9 & 36 \\
\hline 0.68 & 2200 & 3.798 & 0.012 & 0.9 & 36 \\
\hline 0.69 & 2040 & 3.784 & 0.011 & 0.9 & 36 \\
\hline 0.7 & 1890 & 3.772 & 0.011 & 0.9 & 37 \\
\hline 0.71 & 1780 & 3.759 & 0.01 & 0.9 & 37 \\
\hline 0.72 & 1680 & 3.748 & 0.01 & 0.9 & 37 \\
\hline 0.73 & 1540 & 3.737 & 0.009 & 0.8 & 37 \\
\hline 0.74 & 1420 & 3.727 & 0.008 & 0.8 & 37 \\
\hline 0.75 & 1310 & 3.717 & 0.008 & 0.8 & 37 \\
\hline 0.76 & 1190 & 3.708 & 0.007 & 0.8 & 37 \\
\hline 0.77 & 1100 & 3.699 & 0.007 & 0.8 & 37 \\
\hline 0.78 & 1030 & 3.691 & 0.006 & 0.8 & 37 \\
\hline 0.79 & 928 & 3.683 & 0.006 & 0.8 & 38 \\
\hline 0.8 & 850 & 3.675 & 0.005 & 0.8 & 40 \\
\hline 0.81 & 775 & 3.668 & 0.005 & 0.8 & 41 \\
\hline 0.82 & 707 & 3.661 & 0.005 & 0.8 & 42 \\
\hline 0.83 & 647 & 3.654 & 0.004 & 0.7 & 44 \\
\hline 0.84 & 590 & 3.647 & 0.004 & 0.7 & 45 \\
\hline 0.85 & 534 & 3.641 & 0.004 & 0.7 & 46 \\
\hline 0.86 & 479 & 3.635 & 0.003 & 0.7 & 47 \\
\hline 0.87 & 431 & 3.63 & 0.003 & 0.7 & 49 \\
\hline 0.88 & 383 & 3.624 & 0.003 & 0.7 & 51 \\
\hline 0.89 & 343 & 3.619 & 0.002 & 0.7 & 52 \\
\hline 0.9 & 303 & 3.614 & 0.002 & 0.7 & 54 \\
\hline 0.91 & 271 & 3.609 & 0.002 & 0.7 & 56 \\
\hline 0.92 & 240 & 3.604 & 0.002 & 0.7 & 57 \\
\hline 0.93 & 209 & 3.6 & 0.002 & 0.7 & 59 \\
\hline 0.94 & 183 & 3.595 & 0.001 & 0.7 & 62 \\
\hline 0.95 & 156 & 3.591 & 0.001 & 0.7 & 65 \\
\hline 0.96 & 134 & 3.587 & 0.001 & 0.7 & 69 \\
\hline 0.97 & 113 & 3.583 & 0.001 & 0.7 & 73 \\
\hline & & & & & \\
\hline 0.93 &
\end{tabular}


Draft for J of Micromanufacturing

\begin{tabular}{|r|r|r|l|r|r|}
\hline 0.98 & 96 & 3.579 & 0.001 & 0.7 & 78 \\
\hline 0.99 & 79 & 3.575 & 0.001 & 0.7 & 83 \\
\hline 1 & 64 & 3.572 & 0.001 & 0.7 & 90 \\
\hline 1.01 & 51.1 & 3.568 & - & 0.7 & 97 \\
\hline 1.02 & 39.9 & 3.565 & - & 0.7 & 105 \\
\hline 1.03 & 30.2 & 3.562 & - & 0.7 & 112 \\
\hline 1.04 & 22.6 & 3.559 & - & 0.6 & 120 \\
\hline 1.05 & 16.3 & 3.556 & - & 0.6 & 135 \\
\hline 1.06 & 11.1 & 3.553 & - & 0.6 & 145 \\
\hline 1.07 & 8 & 3.55 & - & 0.6 & 155 \\
\hline 1.08 & 6.2 & 3.547 & - & 0.6 & 160 \\
\hline 1.09 & 4.7 & 3.545 & - & 0.6 & 165 \\
\hline 1.1 & 3.5 & 3.542 & - & 0.6 & 175 \\
\hline 1.11 & 2.7 & 3.54 & - & 0.6 & 180 \\
\hline 1.12 & 2 & 3.537 & - & 0.6 & 185 \\
\hline 1.13 & 1.5 & 3.535 & - & 0.6 & 190 \\
\hline 1.14 & 1 & 3.532 & - & 0.6 & 200 \\
\hline 1.15 & 0.68 & 3.53 & - & 0.6 & 210 \\
\hline 1.16 & 0.42 & 3.528 & - & 0.6 & 230 \\
\hline 1.17 & 0.22 & 3.526 & - & 0.6 & 260 \\
\hline 1.18 & 0.065 & 3.524 & - & 0.6 & 320 \\
\hline 1.19 & 0.036 & 3.522 & - & 0.6 & 345 \\
\hline 1.2 & 0.022 & 3.52 & - & 0.6 & 355 \\
\hline 1.21 & 0.013 & 3.518 & - & 0.6 & 380 \\
\hline 1.22 & 0.0082 & 3.517 & - & 0.6 & 390 \\
\hline 1.23 & 0.0047 & 3.515 & - & 0.6 & 405 \\
\hline 1.24 & 0.0024 & 3.513 & - & 0.6 & 410 \\
\hline 1.25 & 0.001 & 3.511 & - & 0.6 & 430 \\
\hline 1.26 & 0.00036 & 3.509 & - & 0.6 & 440 \\
\hline 1.27 & 0.0002 & 3.508 & - & 0.6 & 455 \\
\hline 1.28 & 0.00012 & 3.506 & - & 0.6 & 470 \\
\hline 1.29 & 0.000071 & 3.505 & - & 0.6 & 500 \\
\hline 1.3 & 0.000045 & 3.503 & - & 0.6 & 525 \\
\hline 1.31 & 0.000027 & 3.502 & - & 0.6 & 550 \\
\hline 1.32 & 0.000016 & 3.5 & - & 0.6 & 580 \\
\hline 1.33 & 0.000008 & 3.499 & - & 0.6 & 610 \\
\hline 1.34 & 0.0000035 & 3.497 & - & 0.6 & 650 \\
\hline 1.35 & 0.0000017 & 3.496 & - & 0.6 & 670 \\
\hline 1.36 & 0.00000095 & 3.495 & - & 0.6 & 675 \\
\hline 1.37 & 0.0000006 & 3.494 & - & 0.6 & 680 \\
\hline 1.38 & 0.00000038 & 3.492 & - & 0.6 & 685 \\
\hline 1.39 & 0.00000023 & 3.491 & - & 0.6 & 690 \\
\hline 1.4 & 0.00000014 & 3.49 & - & 0.6 & 700 \\
\hline 1.41 & $8.5 \mathrm{E}-08$ & 3.489 & - & 0.6 & 710 \\
\hline & & & & & \\
\hline
\end{tabular}


Draft for J of Micromanufacturing

\begin{tabular}{|r|r|r|l|r|r|}
\hline 1.42 & 0.00000005 & 3.488 & - & 0.6 & 720 \\
\hline 1.43 & $2.5 \mathrm{E}-08$ & 3.487 & - & 0.6 & 730 \\
\hline 1.44 & $1.8 \mathrm{E}-08$ & 3.486 & - & 0.6 & 740 \\
\hline 1.45 & $1.2 \mathrm{E}-08$ & 3.485 & - & 0.6 & 750 \\
\hline
\end{tabular}

